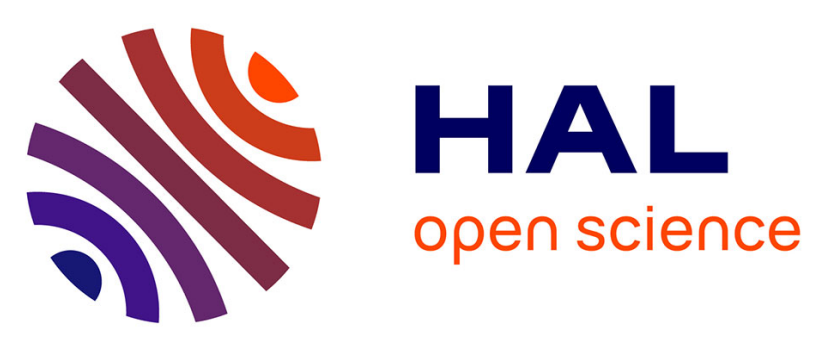

\title{
Saturated Laser-Induced Fluorescence in liquid kerosene seeded with a dye: influence of temperature and excitation intensity
}

Pierre Doublet, Christine Lempereur, Mikael Orain, Virginel Bodoc, Pierre Gajan

\section{To cite this version:}

Pierre Doublet, Christine Lempereur, Mikael Orain, Virginel Bodoc, Pierre Gajan. Saturated LaserInduced Fluorescence in liquid kerosene seeded with a dye: influence of temperature and excitation intensity. Applied Physics B - Laser and Optics, 2019, 125 (186), pp.1-8. 10.1007/s00340-019-7299-4 . hal-02299111v2

\section{HAL Id: hal-02299111 \\ https://hal.science/hal-02299111v2}

Submitted on 29 Nov 2019

HAL is a multi-disciplinary open access archive for the deposit and dissemination of scientific research documents, whether they are published or not. The documents may come from teaching and research institutions in France or abroad, or from public or private research centers.
L'archive ouverte pluridisciplinaire HAL, est destinée au dépôt et à la diffusion de documents scientifiques de niveau recherche, publiés ou non, émanant des établissements d'enseignement et de recherche français ou étrangers, des laboratoires publics ou privés. 


\title{
Saturated Laser-Induced Fluorescence in liquid kerosene seeded with a dye: influence of temperature and excitation intensity
}

\author{
P. DOUBLET ${ }^{1,2}$, C. LEMPEREUR ${ }^{2}$, M. ORAIN ${ }^{2 *}$, V. BODOC ${ }^{2}$, P. GAJAN ${ }^{2}$ \\ ${ }^{1}$ SAFRAN AIRCRAFT ENGINES, 77550 Moissy-Cramayel, France \\ ${ }^{2}$ ONERA - The French Aerospace Lab, 31000 Toulouse, France
}

Rapid communication

\section{STATEMENT}

The content of this manuscript submitted to "Applied Physics B" is unpublished material that is not being submitted for publication elsewhere.

${ }^{*}$ Corresponding author: Pierre DOUBLET

ONERA - The French Aerospace Lab

E-mail address: pierre.doublet@onera.fr 


\section{ABSTRACT}

Fluorescence-based techniques are commonly implemented to characterize droplet sprays in flows at temperature above $293 \mathrm{~K}$. Nevertheless, in the case of gas turbine altitude relight, aeroengines are exposed to critical operating conditions where the liquid fuel temperature can reach values as low as $40^{\circ} \mathrm{C}$. As a result, it is necessary to address the influence of this parameter on fluorescence intensity for temperatures below room values. In this paper, an innovative SLIF (Saturated Laser-Induced Fluorescence) excitation scheme is proposed in order to overcome the strong temperature dependence of fluorescence in liquid kerosene seeded with a dye. The findings of this work are based on gated sequential images recorded with an intensified camera during and after the laser pulse duration so as to sample the temporal evolution of the fluorescence emission. The results show that, for low laser excitation intensity, the fluorescence intensity remains proportional to the laser power. However, a non-linear response is observed in the case of high excitation intensity. This difference can be used to limit the fluorescence dependence on temperature by carefully selecting the temporal window where fluorescence is detected. Finally, although the method presented in this paper is applied to cold liquid kerosene, its range of application can be extended to other liquids and other temperature ranges.

Keywords: fluorescence, temperature, saturation, liquid phase

\section{Introduction}

Droplet spray characterization often relies on laser techniques and image processing that provide information such as droplet size, velocity and concentration. In the literature, experimental investigations of droplets have been performed at ambient and / or high temperatures and pressures $[1,2]$ although there is a constant need for additional data under such harsh conditions to help validate numerical simulations. However, no such investigations have been conducted for altitude relight conditions of aircraft engines (low fuel temperature and sub-atmospheric pressure). Moreover, the 
combination of reduced temperatures and pressures considerably increases certain physical properties, such as surface tension and viscosity, thus altering spray atomization [1,3]. In these conditions, the fuel injector generates larger droplets, liquid fuel evaporation is limited and consequently spray ignition is more difficult. Fine characterization of spray properties in these challenging conditions is needed to help engine manufacturers improve injector design and ultimately ignition performances. Among the different spray characterization techniques, Planar Droplet Sizing (PDS) became popular in the last decade because of its ability to yield an instantaneous 2D-map of droplet Sauter mean diameter: this technique greatly reduces data acquisition time compared to Phase Doppler Anemometry (PDA) [4,5]. This method is based on the ratio between laser-induced fluorescence and Mie scattering images [6]. Until now, most of the work on PDS was carried out in water sprays using different types of dye. Domman et al [7] and Chaze et al. [8] used Rhodamine as a dye in their experiments while Pyranine was added to water in the experiments by Mishra et al [9]. Other dyes such as fluorescein with water as a solvent were used in the Chaze experiments. Jermy et al. [10] implemented the PDS technique in a cooling spray that was too dense for PDA measurements by using sodium salicylate as the dye mixed with water. Some work was also performed with biofuels such as ethanol. For example, Lempereur et al. [4] implemented the PDS technique to an ethanol spray seeded with rhodamine 6G. Koegl [11] studied LIF and Mie signals from an ethanol micrometric droplet as a function of its diameter, with eosin as a dye. Eosin was also used to study ethanol and butanol sprays with the two-phase structured laser illumination planar imaging droplet sizing [12]. A relatively small number of studies were performed on realistic fuels and these were mainly dedicated to automotive fuels. For example, tetramethyl-p-phylene diamine (TMPD) was used by Kamimoto et al. [6] and Yeh et al. [13] and 3 pentanone was added to iso-octane fuel by Stojkovic et al. [14]. Regarding applications of PDS to aviation fuels, very few publications could be found in the literature. Le Gal et al. [15] used p-Terphenyl (PTP) as a dye in mineral spirit (kerosene without its fluorescing components), at ambient temperature and pressure. This suggests a need to develop the PDS technique for kerosene fuel under challenging operating conditions such as altitude relight. The current paper aims at providing a contribution in the field of Planar Droplet Sizing measurements applied to realistic aviation fuels. 


\section{Theoretical background}

Fluorescence intensity depends on several parameters and can be expressed as follows:

$I_{f}(T) \sim I_{0} \epsilon(T) \Phi_{f}(T) C$

where $I_{0}$ is the incident laser intensity, $C$ the concentration of the fluorescent species, $\Phi_{f}$ its quantum yield $(Q Y)$ and $\epsilon$ its absorption cross section. The last two parameters depend on the fluorescent specie considered. For example, Chaze et al. [8] studied the influence of temperature on the fluorescence of several dyes and reported different temperature behaviours. On one hand, fluorescence increases with temperature for fluorescein disodium because of an increase in the absorption cross section. By contrast, fluorescence decreases for rhodamine 6G due to the decrease of the $Q Y$ as temperature increases (quenching effect). The solvent containing the dye can be an additional parameter that also affects the temperature dependence of the dye fluorescence.

As a first assumption for Planar Droplet Sizing, the fluorescence intensity emitted by a droplet $I_{f}$ is proportional to its volume [6]. Nonetheless, if the dye concentration is high or if the absorption cross section is large, this assumption needs to be re-evaluated. In such a case, droplet fluorescence intensity can then be written as $[7,16]$ :

$I_{f}=\mathrm{a}_{\mathrm{f}} d^{F(C)}$

where $a_{f}$ is a constant, $d$ the droplet diameter and $F$ a parameter which depends on the dye concentration $C$. For low absorption, $F(C)$ is close to 3 (i.e. the theoretical value), but strongly decreases as absorption increases [16]. Moreover, as can be seen in equation (1), the quantum yield $\Phi_{f}$ and the absorption cross section $\epsilon$ depend on temperature. In some cases, this temperature dependence can be high which alters the reliability of the PDS technique [8].

A way of limiting the quenching effect consists in working with saturated laser-induced fluorescence. This method is used in particular to measure $\mathrm{OH}$ or NO species concentrations in flames at high pressure $[17,18]$. However, to the best of the authors' knowledge, the saturated laser- induced fluorescence is mainly applied to gases and the only study conducted in liquids is that of [8]. 
In the present paper, the saturated laser-induced fluorescence method is carried out in liquid kerosene in order to overcome the temperature dependence of the dye added to the fuel. This allows to perform accurate Planar Droplet Sizing measurements under challenging conditions, which is the ultimate goal of this study.

\section{Experimental setup and measurement protocol}

The tests are carried out in a calibration cell in order to analyze the influence of temperature and excitation intensity on the fluorescence measurements. Pyrromethene 597-8C9 (PM from now on) is used as a dye in liquid kerosene because of its high miscibility with kerosene, ethanol, methanol and oils [19].

By contrast, despite having useful fluorescence properties as a dye for PDS applications with water, rhodamine 6G cannot be used with kerosene due to non-miscibility between these two compounds. PM concentration is fixed to $1.2 \mathrm{mg} / \mathrm{L}$ in order to minimize absorption along the laser path. This value, which was carefully selected from a parametric investigation of PM fluorescence signal versus concentration, is sufficient to study realistic kerosene sprays. The seeded liquid is placed into a 10 mm-size quartz test cell from HELLMA, and then introduced into a Luma 40 cuvette holder from Quantum Northwest in order to regulate its temperature between -30 and $50{ }^{\circ} \mathrm{C}$.

A frequency-doubled Nd:YAG pulsed laser (Big Sky, Quantel) emitting light at $532 \mathrm{~nm}$ is used to excite fluorescence. An energy meter is employed to quantify the pulse-to-pulse variations of the laser energy. The beam is then converted into a collimated sheet passing through the cell. A spectrometer (HR2000+, Ocean Optics) and an intensified camera (PIMAX 4, Gen III, Roper Scientific) are placed at $90^{\circ}$ from the laser propagation, on opposite sides of the test cell, in order to perform simultaneous fluorescence measurements. A long-pass edge filter (BLP01-532R, Semrock) enables the acquisition of the fluorescence signal close to the laser line with a deep laser-line blocking (Fig. 1.).

Both measurement devices record the fluorescence above the excitation wavelength $(\lambda>540 \mathrm{~nm})$ spatially integrated over a small area $\left(10 \times 10 \mathrm{~mm}^{2}\right)$. The transmission of the high-pass filter has a flat profile above $542 \mathrm{~nm}$ with values in excess of 99\% and therefore does not require any correction. 
However, the data cannot be quantitatively compared. The first reason is that the spectrometer provides calibrated spectral data whereas the camera spectrally integrates the fluorescence without correction for its own spectral response. Additionally, both devices have different measurement sensitivities. Nevertheless, the data can be compared after normalization as shown later in the text.

The interesting feature lies in the acquisition modes of the intensified camera. The gate width (i.e. the integration time) can generally be chosen to include all the duration of the fluorescence emission with a constant delay with respect to the laser pulse (Fig. 2. (a)). However, the gate width can also be set to a smaller value (here $3 \mathrm{~ns}$ ) and moved gradually at increasing delays after the laser pulse in order to measure the temporal evolution of fluorescence (Fig. 2. (b)). Typically, a few tens of pulses are necessary to describe the time evolution of PM fluorescence. In this acquisition mode, the laser intensity has to remain stable over a few shots. The measurement protocol was fixed as follows: the laser is switched on for a few tens of seconds without hitting the cell, until its intensity is stabilized (rms fluctuation $<5 \%$ ). The test cell is then illuminated during the required number of pulses only, in order to minimize the photodamage of PM. Actually, after this short laser exposure, the fluorescence level does not recover its initial value because of bleaching. As a result, the fluid is changed in the test cell after each measurement sequence.

\section{Influence of temperature on the fluorescence of PM-seeded kerosene}

The temperature conditions for altitude relight of aeroengines engines range from -40 to $0{ }^{\circ} \mathrm{C}$. However, a wider exploration has been conducted, up to $50^{\circ} \mathrm{C}$, which can be useful for other purposes.

The objective is to quantify the temperature sensitivity of PM fluorescence to define the conditions in which the variations of this parameter can be considered as negligible on the fluorescence measurement.

Measurements are first conducted with a low laser energy chosen to yield a linear fluorescent emission during the whole pulse duration. The emission spectrum, measured above $540 \mathrm{~nm}$, is broadband with a maximum at $600 \mathrm{~nm}$. For each temperature, the corresponding spectrum is normalized by the maximum value of the spectrum at $-28{ }^{\circ} \mathrm{C}$. Fluorescence quenching is observed when temperature 
increases (Fig. 3). The profiles normalized by their maximum value (not shown here) do not exhibit any noticeable shape difference (e.g, redshift) with the wavelength.

The integration of the fluorescence signal over the emission spectral range is plotted as a function of temperature in Fig. 4. The measurement was also performed with the camera, using a gate width of 50 ns to include all the fluorescence emission duration. Both curves, normalized by their value at $\mathrm{T}=0$ ${ }^{\circ} \mathrm{C}$, are in good agreement. In order to quantify the temperature dependence of the dye emission, a sensitivity coefficient s [8] can be introduced:

$I_{f}(T)=I_{f}\left(T_{0}\right) e^{-s\left(T-T_{0}\right)}$

where $T_{0}$ is an arbitrary temperature, chosen here as $T_{0}=0{ }^{\circ} \mathrm{C}$, and $s$ is estimated at $-1.3 \% /{ }^{\circ} \mathrm{C}$, which can be compared to that of rhodamines. For example, Rh6G is often chosen for its low temperature sensitivity $\left(s(R h 6 G)=-0.07 \% /{ }^{\circ} \mathrm{C}\right)$ enabling temperature-independent fluorescence measurements, while $\mathrm{RhB}$ is much more sensitive $\left(s(R h B)=-2.3 \% /{ }^{\circ} \mathrm{C}\right)$ and can be used for temperature measurements in liquids [8]. The sensitivity of PM lies between that of these two rhodamines and cannot be neglected since, for instance, the effect of the temperature on Pyrromethene fluorescence measurement can cause a reduction of $40 \%$ between $-30{ }^{\circ} \mathrm{C}$ and $0{ }^{\circ} \mathrm{C}$.

\section{Influence of the laser intensity: saturation of fluorescence}

When pulsed lasers are used to excite fluorescence, their irradiance usually exceeds the saturation intensity of common fluorescent dyes. The fluorescence signal therefore loses its linear dependence on the laser irradiance [8]. This phenomenon will later be illustrated by the temporal profiles of fluorescence for increasing laser intensity. The laser irradiance is regulated via the QSwitch / Flash Lamp internal delay $\Delta$. The maximum irradiance value, approximately $6 \times 10^{6} \mathrm{~W} / \mathrm{cm}^{2}$, is obtained for $\Delta_{0}=176 \mu$ s and decreases for increasing delays. The data used to estimate irradiance is affected by some uncertainties due to the estimation of the temporal profile of the laser pulse and spatial intensity profile of the laser sheet. The temporal evolution of the laser pulse is measured by using a band pass filter (BP 532/25, Lavision) placed in front of the camera. Given the FWHM (i.e. $25 \mathrm{~nm}$ ) of the filter, a fluorescence signal is also acquired by the camera but this signal is negligible compared to the one 
emitted by the laser. The sequential acquisition mode of the camera is used to obtain the temporal evolution of the laser pulse. For the delay used in the previous paragraph $\left(\Delta_{2}=320 \mu\right.$ s, which corresponds to a laser irradiance of $2 \times 10^{6} \mathrm{~W} / \mathrm{cm}^{2}$ ), fluorescence intensity increases with the laser energy and then decreases with a time shift induced by the fluorescence lifetime. On the other hand, when the energy is higher $\left(\Delta_{1}=300 \mu\right.$ s, approximately $\left.3 \times 10^{6} \mathrm{~W} / \mathrm{cm}^{2}\right)$, a saturation clearly appears, as shown in Fig. 5 (see the dashed black marks). In this case, fluorescence intensity remains constant until the laser energy is lower than a given excitation threshold.

The fluorescence saturation phenomenon can also be visualized on the graph of fluorescence intensity versus excitation intensity of the laser (Fig. 6). The lower part of each curve follows the rising edge of the laser pulse whereas the upper part represents the falling edge. The fluorescence lifetime is illustrated by the fact that, for a given excitation intensity, the fluorescence intensity is greater on the falling edge. In the non-saturated case, fluorescence intensity increases with the excitation up to its maximum value and then decreases. In the saturated case, the fluorescence intensity reaches its maximum value when the excitation intensity is equal to $60 \%$ of the maximum excitation intensity in this case. For higher excitation intensities, a saturation of the fluorescence intensity is also observed.

This saturation phenomenon is interesting and can be exploited to suppress the temperature dependence of PM fluorescence for measurements in sprays. Once the laser intensity threshold is reached, the fluorescence intensity remains constant for the length of time during which the laser irradiance is larger than this threshold value, regardless of the temperature.

The temporal evolution of fluorescence signal with temperature is presented in Fig.7. Three measurement timings can be analyzed to illustrate the saturation behaviour. The first one, labelled (a), shows that if the fluorescence signal is integrated over the non-linear part of the curve, between 70 and 80 ns (blue diamonds in Fig. 8) then it is temperature independent. Next, for measurement timing (b), if fluorescence is integrated over the falling edge, when the phenomenon becomes linear again between 85 and 105 ns (green triangles in Fig.8), then the fluorescence emission exhibits the same decrease with temperature as in Fig. 4. In particular, the fluorescence lifetime also depends on temperature for molecules having a temperature-dependent quantum yield. Finally, for measurement timing (c), if fluorescence is integrated over its full duration (red dots in Fig.8) then the measured 
emission is globally less dependent upon temperature. This decrease in dependence is due to the fact that the first temporal part of the curves, which is temperature independent, is integrated in the measurement. A summary of these results is presented in Fig. 8.

The time range in which fluorescence intensity is integrated is a key parameter. Measurement accuracy strongly depends on the acquisition timing of the camera. For example, Fig. 8 shows that for the minimum and maximum temperatures (i.e. $-28{ }^{\circ} \mathrm{C}$ or $50{ }^{\circ} \mathrm{C}$ here), the difference between the measurements carried out in the temperature dependent and independent regions, can reach up to \pm 30

\%. This shows that it is extremely important to know exactly in which regime (e.g. temperature dependent or independent) measurements are performed, to obtain reliable results in future PDS applications. Finally, it is important to note that this methodology using gated acquisitions is only possible with an intensified camera. If the PDS technique is carried out with conventional CCD or CMOS cameras, the gate time cannot be adjusted to fulfill this requirement.

\section{Perspectives: Extension to a laser sheet for planar measurements}

In the present case, a parallel light sheet with homogeneous fluence within the field of view $(10 \times 10$ $\mathrm{mm}^{2}$ ) is obtained with a collimator placed at the exit of the laser. For planar measurements such as Planar Droplet Sizing, the field of view is about $100 \times 100 \mathrm{~mm}^{2}$ and the laser sheet spatial distribution can introduce a measurement bias. The spatial distribution of the laser sheet intensity exhibits a Gaussian profile. As a result, fluorescence saturation can occur for the droplets located in the center of the laser sheet, ie. with the highest energy, as opposed to the droplets located in the laser sheet edges. This aspect is currently under investigation.

\section{Conclusion}

This study shows the influence of temperature on the fluorescence of liquid kerosene seeded with Pyrromethene as a dye. Fluorescence measurements carried out under temperatures ranging from -30 to $50{ }^{\circ} \mathrm{C}$. exhibit a temperature sensitivity of about $-1.3 \% /{ }^{\circ} \mathrm{C}$. This strong temperature dependence is a drawback to perform fluorescence measurements in sprays (i.e. Planar Droplet Sizing). Nonetheless, an image acquisition strategy is proposed to overcome this issue. For low excitation intensity, the 
fluorescence intensity response is proportional to the laser excitation intensity, whereas a non-linear fluorescence response is observed for high excitation intensity. Fluorescence saturation clearly appears for a given laser intensity threshold above which fluorescence intensity is independent of temperature (in the range $\left[-30,50{ }^{\circ} \mathrm{C}\right]$ ). This effect can be advantageous for experiments conducted under cold conditions, for example in high-altitude relight experiments where the temperature is low. The development of optical diagnosis under these conditions seems useful for the characterization of aeronautical injection systems.

\section{Acknowledgements}

This work is conducted between ONERA and SAFRAN AIRCRAFT ENGINES. The authors gratefully acknowledge the ANRT (Association Nationale de la Recherche et de la Technologie) which supports part of the PhD.

\section{REFERENCES}

[1] A. H. Lefebvre, “Atomization and Sprays”, Hemisphere publishing corporation, 1989.

[2] X. F. Wang and A. H. Lefebvre, "Influence of ambient air pressure on pressure swirl atomization”, $32^{\text {nd }}$ ASME International Gas Turbine Conference, 1987.

[3] S. Yao, T. Zhang et T. Fang, "Effect of viscosities on structure and instability of sprays from a swirl atomizer”, Exp. Therm. Fluid Science, 2012, vol. 39, 158-166.

[4] C. Lempereur, V. Bodoc, "Etude de la technique de granulométrie Planar Droplet Sizing sur un brouillard issu d'un injecteur aéronautique”. 16 ${ }^{\text {ème }}$ Congres Francophone de Techniques Laser. 2016.

[5] G. Charalampous et Y. Hardalupas, "Method to reduce errors of droplet sizing based on the ratio of fluorescent and scattered light intensities (laser induced fluorescence/Mie technique)”, Applied Optics, 2011, 50 (20), 3622-3637.

[6] T. Kamimoto, "Diagnostic of transient sprays by means of laser sheet techniques", in COMODIA, 1994, 33-41. 
[7] R. Domann, Y. Hardalupas and A. R. Jones, “A study of the influence of absorption on the spatial distribution of fluorescence intensity within large droplets using Mie theory, geometrical optics and imaging experiments, Measurement Science and Technology, 2002, 13 (3), p.280.

[8] W. Chaze, O. Caballina, G. Castanet, F. Lemoine. "The saturation of the fluorescence and its consequences for laser-induced fluorescence thermometry in liquid flows”. Exp in Fluids. April 2016. $57: 58$.

[9] Y. N. Mishra, E. Kristensson, E. Berrocal, "Reliable LIF/Mie droplet sizing in sprays using structured laser illumination planar imaging”, Optics Express, 2014, 22 (4), 4480-4492.

[10] M. C. Jermy and D. A. Greenhalgh, "Planar dropsizing by elastic and fluorescence scattering in sprays too dense for phase Doppler measurement”, Applied Physics B, 2000, 71 (5), 703-710.

[11] M. Koegl, B. Hofbeck, K. Baderschneider, Y. N. Mishra, F. J. T. Huber, E. Berrocal, S. Will and L. Zigan, “Analysis of LIF and Mie signals from single micrometric droplets for instantaneous droplet sizing in sprays”, Optics Express, 2018, 26 (24), 31750-31766.

[12] M. Koegl, Y. N. Mishra, M. Storch, C. Conrad, E. Berrocal, S. Will and L. Zigan, “Analysis of ethanol and butanol direct-injection sprak-ignition sprays using two-phase structured laser illumination planar imaging droplet sizing, International journal of spray and combustion dynamics, 2018, DOI : 10.1177/I756827718772496.

[13] C-N Yeh, H. Kosaka and T. Kamimoto, "Fluorescence/scattering image technique for particle sizing in unsteady diesel spray, 1993, 93, 4008-4013.

[14] B. D. Stojkovic, V. Sick, "Evolution and impingement of an automotive fuel spray investigated with simultaneous Mie/LIF techniques”, Applied Physics B, 2001, 73, 75-83.

[15] P. Le Gal, N. Farrugia, D. A. Greenhalgh, "Laser sheet dropsizing of dense sprays”, Optics \& Laser technology, 1999, 31, 75-83.

[16] G. Charalampous et Y. Hardalupas, "Numerical evaluation of droplet sizing based on the ratio of Fluorescent and scattered light intensities (lif/mie technique)”, Applied Optics, 2011, 50, 1197-1209.

[17] S. V. Naik, N. M. Laurendeau, "Laser-saturated and linear laser-induced fluorescence measurements of nitric oxide in counterflow diffusion flames under non-sooting oxygen-enriched conditions”, Combustion Science and Technology, 174 (Issue 2), 2002, 1-21. 
[18] P. Desgroux, E. Domingues, M. J. Cottereau, “Measurements of $\mathrm{OH}$ concentrations in flames at high pressure by two-optical path laser induced fluorescence”, Applied Optics, 31, 15, 1992.

[19] D. Sanned, Study of fluorescence dyes for tracing lubrification oil inside marine engines, Master thesis, Lund University, December 2016.

\section{FIGURES}

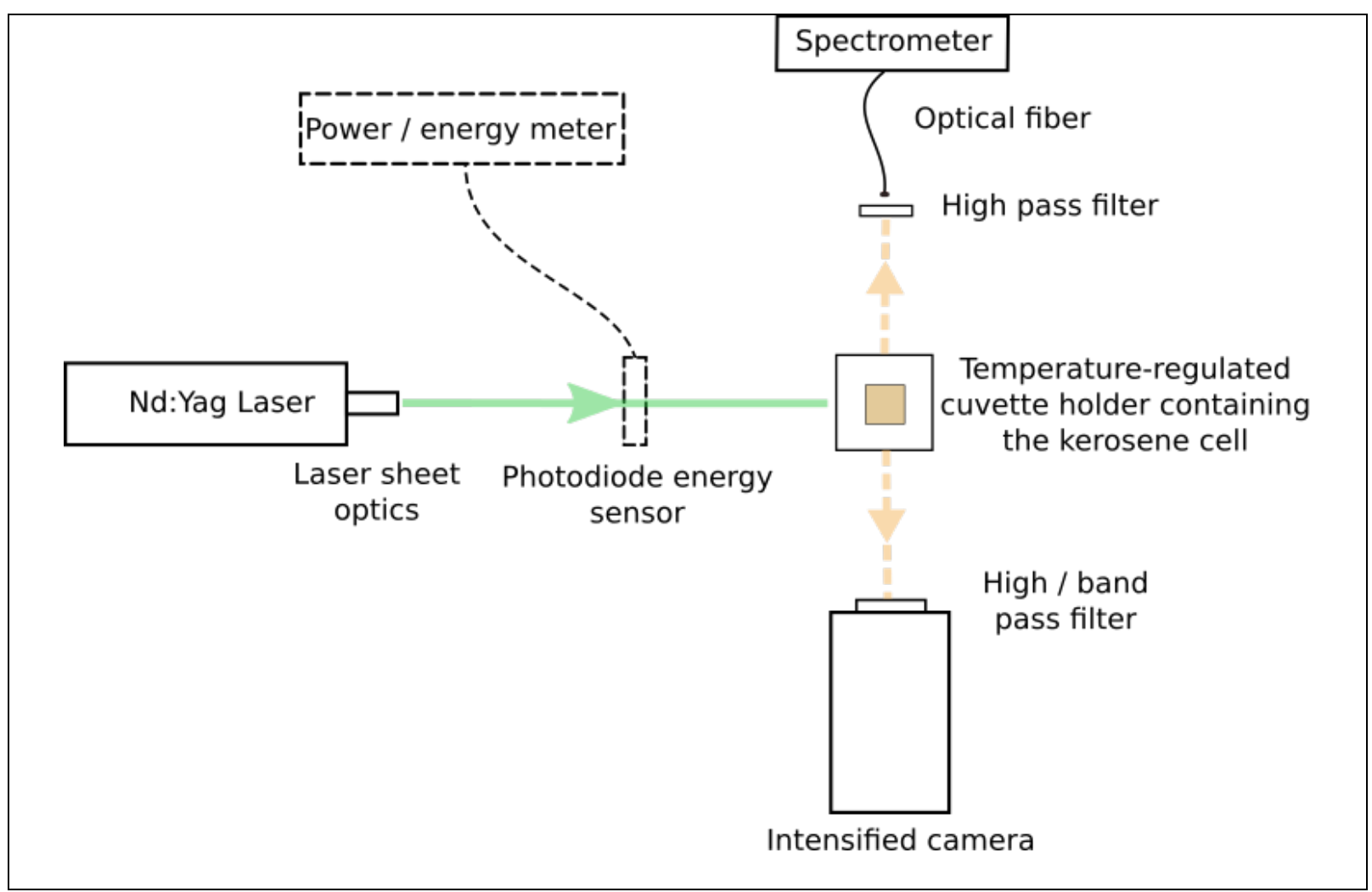

Fig. 1 Experimental set-up (Top view).

(a)

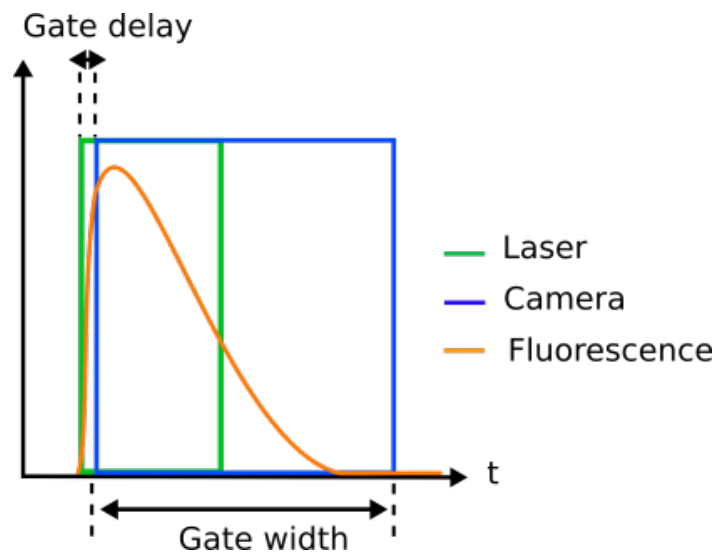


(b)

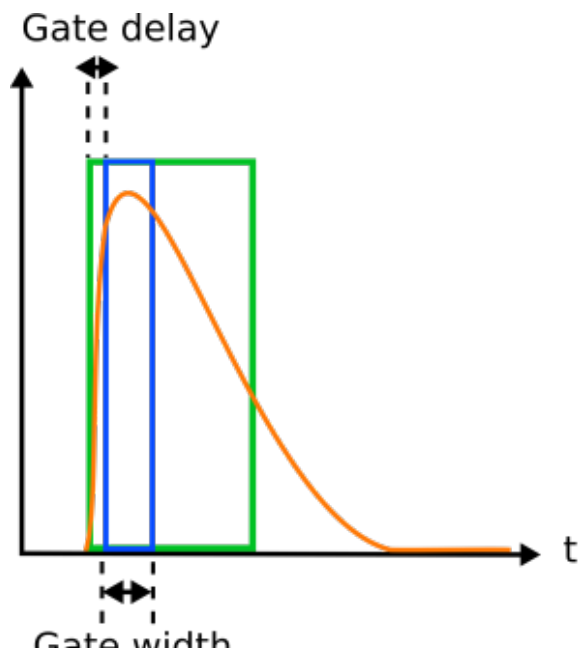

Gate delay

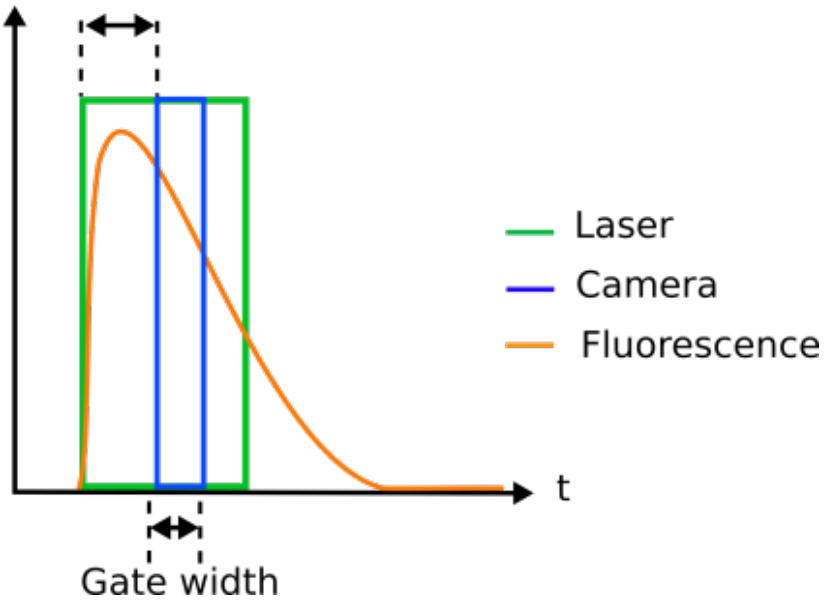

Fig. 2 Repetitive (a) and Sequential (b) acquisition modes of the intensified camera

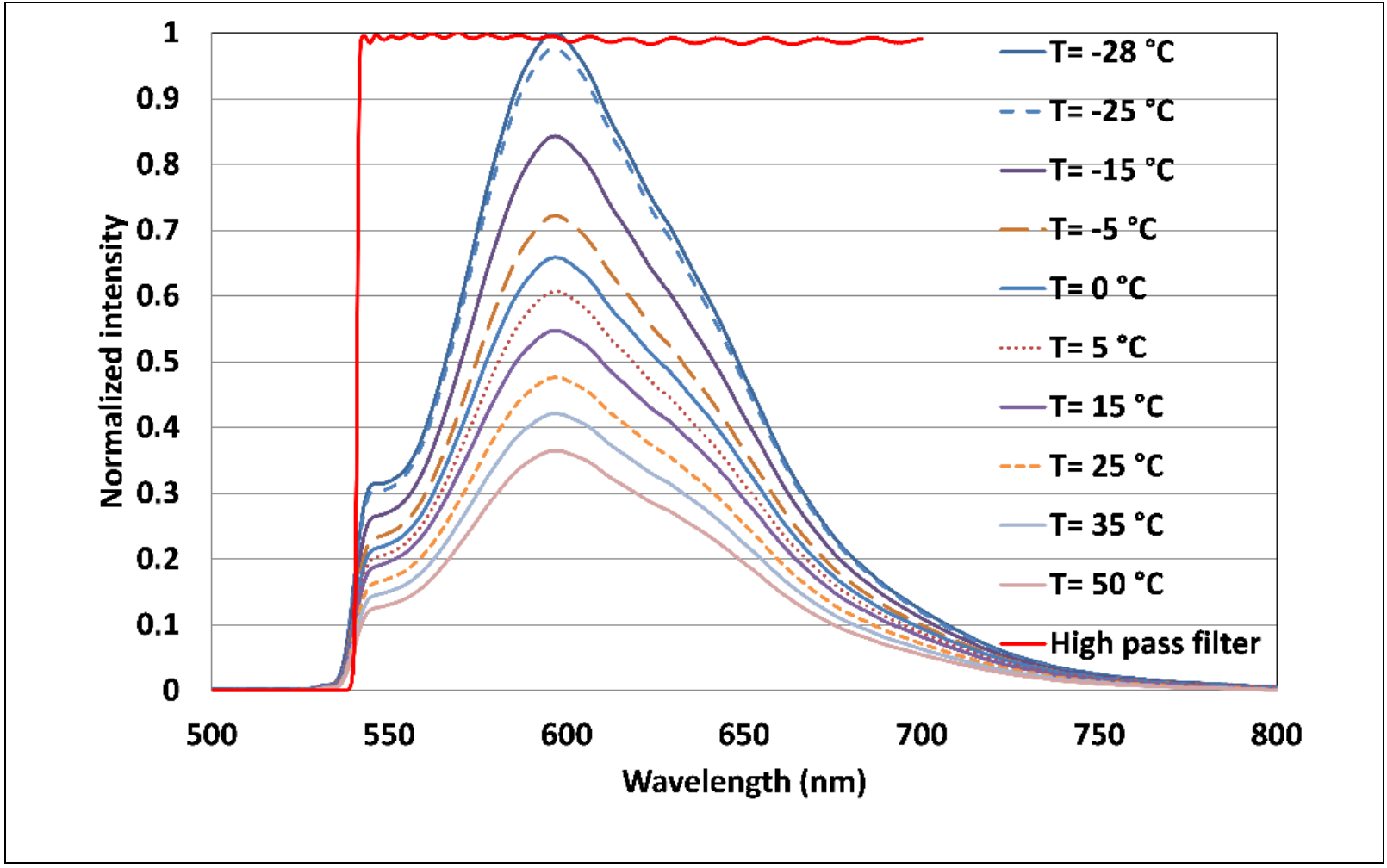

Fig. 3 Evolution of the fluorescence spectrum above $540 \mathrm{~nm}$ with temperature ranging between $-28{ }^{\circ} \mathrm{C}$ and $50{ }^{\circ} \mathrm{C}$. The spectrometer signal is accumulated on three consecutive pulses and averaged over 10 sequences of 3 pulses. The transmission of the high pass filter with a cut-off wavelength at $540 \mathrm{~nm}$ is represented by the red plot. 


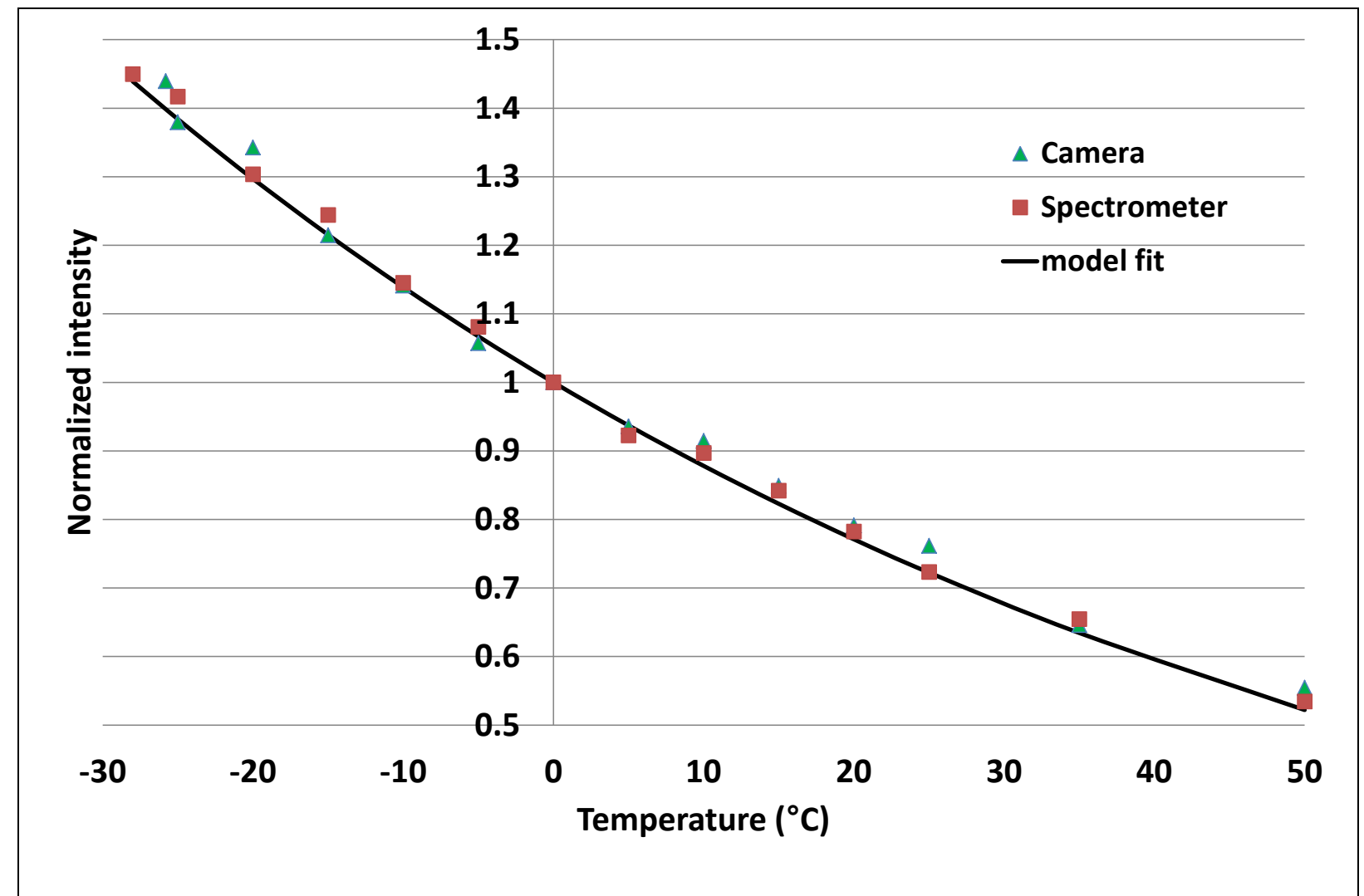

Fig. 4 Evolution of fluorescence intensity with temperature measured by the spectrometer and by the camera. Each signal is normalized by its value at $0{ }^{\circ} \mathrm{C}$. This evolution is observed in the non-saturated case. 


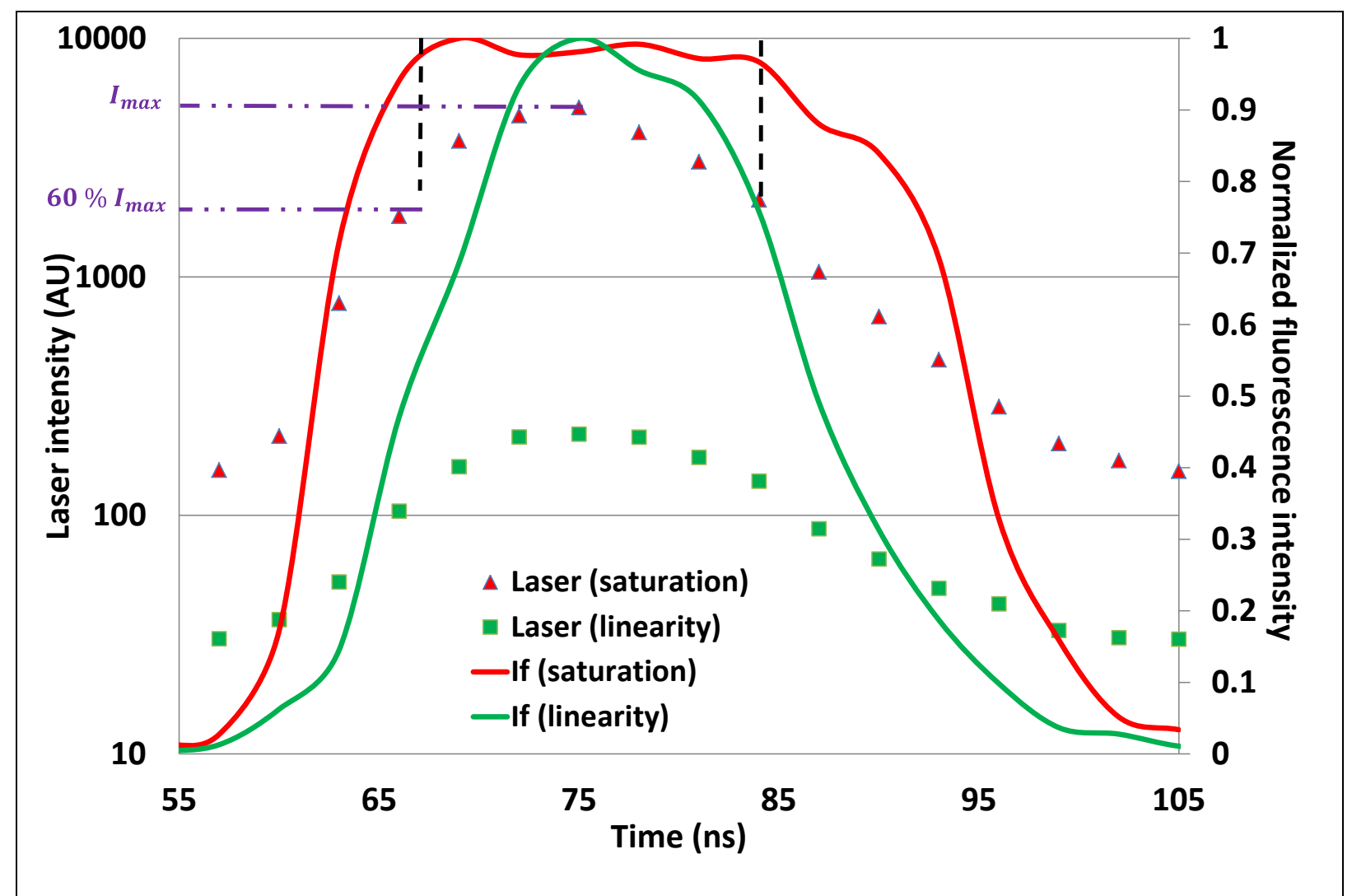

Fig. 5 Temporal evolution of laser energy and fluorescence response for non-saturated case $\left(\Delta_{2}=320\right.$ $\left.\mu \mathrm{s}, 2 \times 10^{6} \mathrm{~W} / \mathrm{cm}^{2}\right)$ and saturated case $\left(\Delta_{1}=300 \mu \mathrm{s}, 3 \times 10^{6} \mathrm{~W} / \mathrm{cm}^{2}\right)$. Each fluorescence signal is normalized by its maximum value over the whole temporal range.

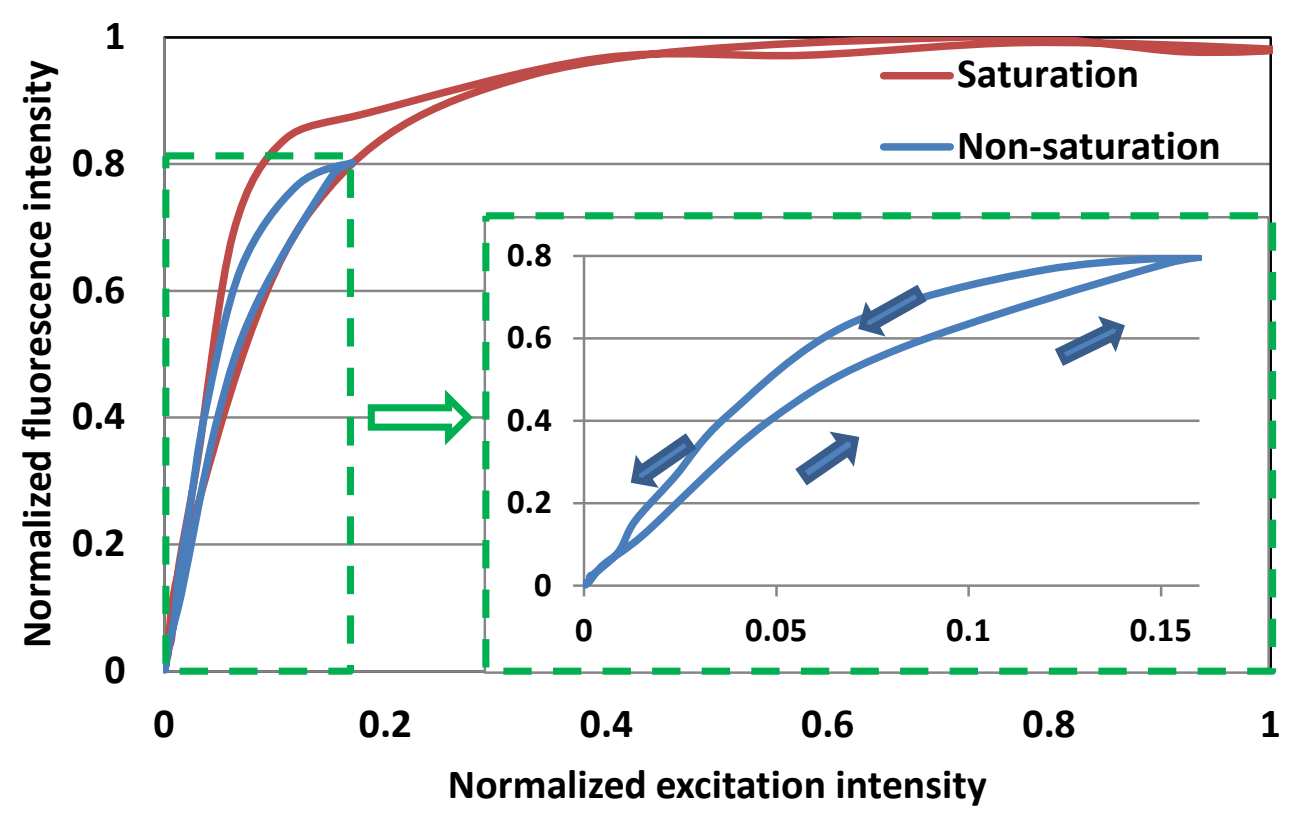


Fig. 6 Normalized fluorescence intensity versus normalized excitation intensity for non-saturated $\left(\Delta_{2}\right.$ $\left.=320 \mu \mathrm{s}, 2 \times 10^{6} \mathrm{~W} / \mathrm{cm}^{2}\right)$ and saturated case $\left(\Delta_{1}=300 \mu \mathrm{s}, 3 \times 10^{6} \mathrm{~W} / \mathrm{cm}^{2}\right)$. Each fluorescence signal is normalized by the maximum value.

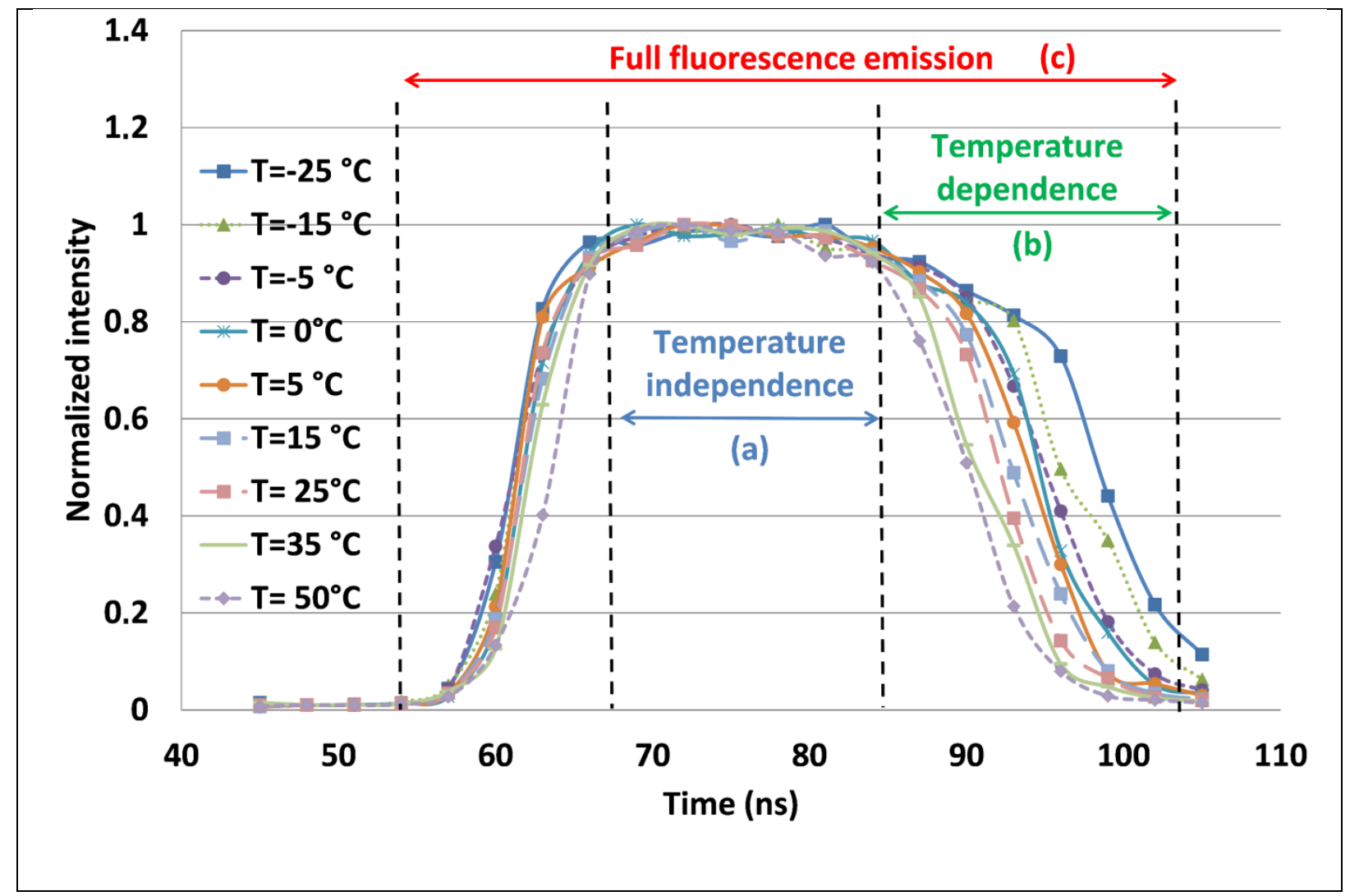

Fig.7 Temporal evolution of fluorescence intensity for various temperatures ranging from -25 to 50 ${ }^{\circ} \mathrm{C}$. Each fluorescence signal is normalized by the global maximum value over the whole temporal and temperature ranges. 


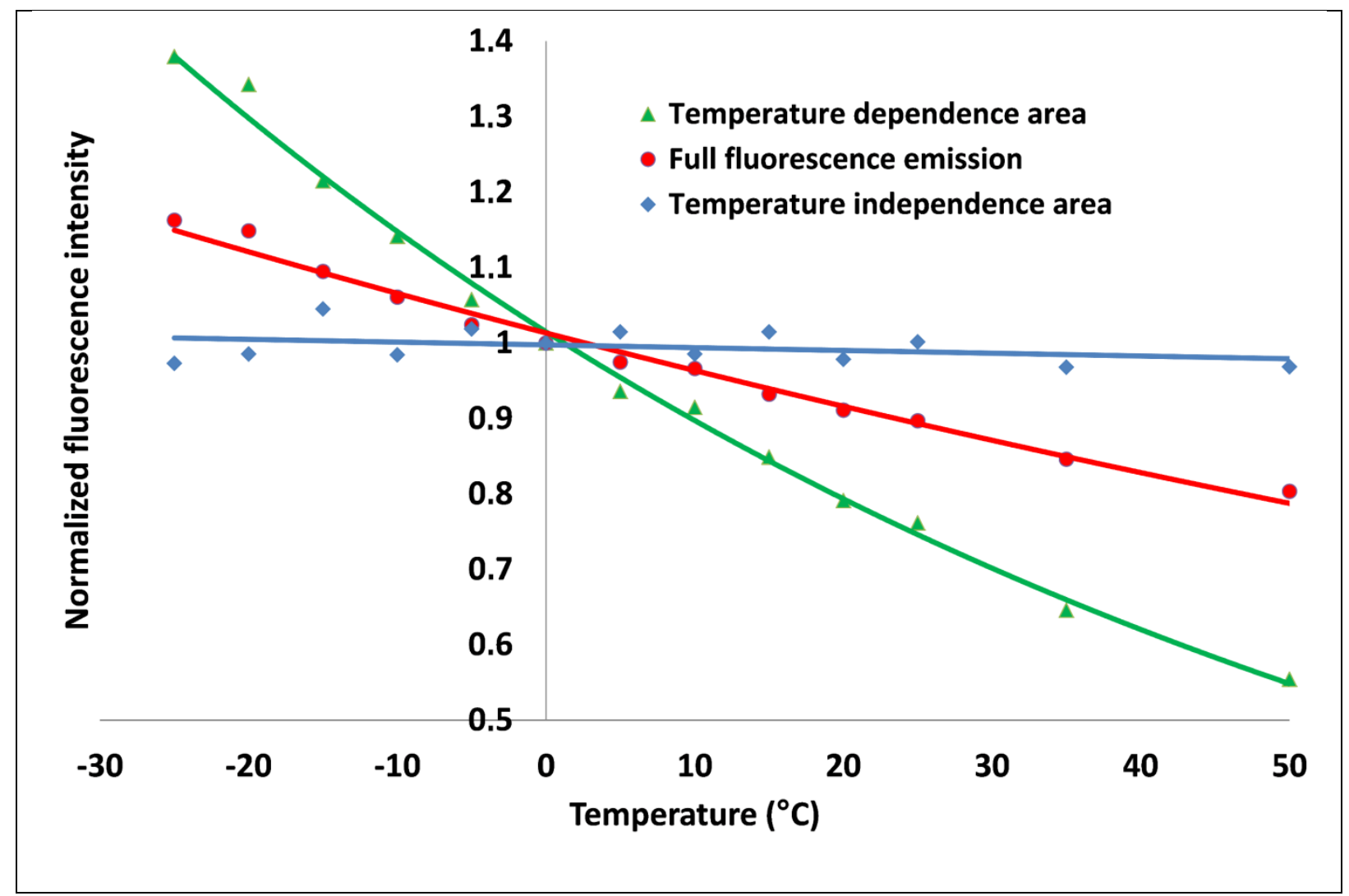

Fig.8 Evolution of fluorescence intensity with temperature measured by the camera for three measurement timings. Each signal is normalized by its value at $0{ }^{\circ} \mathrm{C}$. 